\title{
Chern-Simons modified gravity and closed time-like curves
}

\author{
P. J. Porfírio, J. B. Fonseca-Neto, J. R. Nascimento,
}

A. Yu. Petrov, J. Ricardo ${ }^{1}$ and A. F. Santos ${ }^{2,3, \text { * }}$

${ }^{1}$ Departamento de Física, Universidade Federal da Paraiba

Caixa Postal 5008, 58051-970, João Pessoa, Paraíba, Brazi闭

${ }^{2}$ Instituto de Física, Universidade Federal de Mato Grosso, 78060-900, Cuiabá, Mato Grosso, Brazil

${ }^{3}$ Department of Physics and Astronomy, University of Victoria, 3800 Finnerty Road, Victoria, BC, Canada

\begin{abstract}
We verify the consistency of the Gödel-type solutions within the four-dimensional ChernSimons modified gravity with the non-dynamical Chern-Simons coefficient, for different forms of matter including dust, fluid, scalar field and electromagnetic field, and discuss the related causality issues. We show that, unlike the general relativity, a vacuum solution is possible in our theory. Another essentially new result of our theory having no analogue in the general relativity consists in the existence of the hyperbolic causal solutions for a physically wellmotivated matter.
\end{abstract}

\section{INTRODUCTION}

The studies of the Chern-Simons (CS) modified gravity have a long story. Initially, the CS extension for the three-dimensional gravity has been proposed in [1] as an analogue for the CS extension of quantum electrodynamics which allowed to construct the gauge invariant massive theory. The four-dimensional extension of the gravitational CS term was firstly introduced in [2] within the context of gravitational anomalies, where it was shown to be characterized by an arbitrary pseudo-scalar function $\phi(x)$, that is, the so-called CS coefficient. The new wave of interest to the four-dimensional CS modified gravity, that is, the gravitational model whose action represents itself as a sum of the usual Einstein-Hilbert action and the gravitational CS term, has been inspired by the seminal paper [3], where the four-dimensional gravitational CS term was shown to involve Lorentz symmetry breaking for a special form of the CS coefficient $\phi(x)=$ $k_{\mu} x^{\mu}$, and, evidently, the presence of the gravitational CS term breaks the CPT symmetry for any

* alesandroferreira@fisica.ufmt.br

${ }^{\dagger}$ Electronic address: pporfirio,jfonseca,jricardo,jroberto,petrov@fisica.ufpb.br 
$\phi(x)$. Therefore, the CS modified gravity represents itself as a simplest example of a Lorentz-CPT breaking extension of the general relativity (GR). Also, it was shown in [3] that the Schwarzschild metric continues to be a solution for equations of motion also within CS modified gravity. Further, the gravitational CS term has been discussed as a possible ingredient of the most generic Lorentzbreaking extension of the standard model [4, and it was shown to arise as a quantum correction in the theory describing spinor fields on a curved background [5]. Among other results found for the CS modified gravity in dimensions higher than three, one should also mention the possible higher-dimensional generalizations of the gravitational CS term performed in [6]. Different issues related to Chern-Simons (super)gravities in different dimensions has been discussed [7]. A review on specifics of the CS gravity in four-dimensions has been developed [8].

It is clear that to study the compatibility of any new extended gravity model, in particular, the CS modified gravity, with the observations, one should verify the consistency of the known solutions of the Einstein-Hilbert gravity within the new theory. A wide class of the classical gravitational solutions possessing spherical or axial symmetry has been discussed from this viewpoint in the paper 9. Besides of this, a further generalization of the CS modified gravity has been proposed in that paper, that is, the so-called dynamical Chern-Simons modified gravity where the CS coefficient $\phi(x)$ becomes a dynamical field. This extension opened the possibilities for new studies. In particular, it was showed in [10] that the Kerr metric itself does not solve the modified Einstein equations in this theory, and, to achieve consistency, one should modify some elements of the metric tensor by additive terms depending on the CS coefficient. The cosmological solutions in the dynamical CS modified gravity have been considered in [11].

It is well known that the problem of causality in the gravitational context is strongly polemical. Historically, this problem began to be discussed in [12] where the Gödel metric allowing for the closed time-like curves (CTCs) was found. Further, a very important generalization of the Gödel metric has been introduced, with a number of their properties were studies, in a sequence of papers [13. In particular, it was showed in these papers that in certain cases the CTCs are ruled out for some special relations between constant parameters of this metric. Therefore, it is interesting to verify the consistency of the Gödel-type metric proposed in [13] within the CS modified gravity, especially, to obtain the conditions for parameters allowing to rule out the CTCs. Some preliminary studies in this direction have been performed in [14, where, first, only the case of the original Gödel metric has been considered, second, the results seem to be dependent on the choice of the basis, which naturally calls the interest to solving this problem in terms of the tetrad basis.

Therefore, the natural problem would consist, first, in studying of the compatibility of different 
kinds of the Gödel-type metric (it is known [13] that there are three kinds of this metric, that is, hyperbolic, trigonometric and linear cases) within the CS modified gravity following the procedure used in [13, second, in determining values of the parameters which could rule out the CTC solutions. Namely these problems are considered in this paper.

The structure of the paper looks like follows. In section II, we present a general review of the Gödel-type metrics. Section III is devoted to description of the Gödel-type solutions in the general relativity for different forms of the matter including a relativistic fluid, a cosmological term, scalar and electromagnetic fields, with use of the tetrad basis. In section IV, these solutions are generalized for the CS modified gravity, with the relations between parameters of matter are found explicitly. Finally, in Summary the results are discussed.

\section{GÖDEL-TYPE METRICS}

In this section, we briefly review the Gödel-type metrics consistent with the condition of homogeneity in the space-time (ST-homogeneous), and discuss their classes and causality features related to the existence of closed time-like curves.

In spite of the local validity of the causality principle in general relativity, assured by its local Lorentzian character, it is well known that there are solutions of Einstein field equations displaying causality anomalies given by closed time-like curves. One of the first and best known examples of such a solution is the famous Gödel metric [12]. The rotating Gödel universe belongs to a family of solutions of Einstein equations which are ST-homogeneous, known as Gödel-type metrics [13, whose line element in cylindrical coordinates is given by

$$
d s^{2}=-[d t+H(r) d \theta]^{2}+D^{2}(r) d \theta^{2}+d r^{2}+d z^{2},
$$

and the metric functions $H(r)$ and $D(r)$ obey the following necessary and sufficient conditions for ST-homogeneity

$$
\begin{aligned}
\frac{H^{\prime}(r)}{D(r)} & =2 \omega, \\
\frac{D^{\prime \prime}(r)}{D(r)} & =m^{2},
\end{aligned}
$$

where the prime denotes the derivative with respect to $r$. All the Gödel-type ST-homogeneous metrics are completely characterized by the two parameters $\left(m^{2}, \omega\right)$, where $\omega \neq 0$ is the vorticity, and $-\infty \leq m^{2} \leq \infty$, such that isometric space-times have identical pairs $\left(m^{2}, \omega^{2}\right)$. The Gödeltype ST-homogeneous manifolds admit a $G_{5}$ group of isometries when $0 \leq m^{2}<4 \omega^{2}$, a $G_{7}$ when 
$m^{2}=4 \omega^{2}$ and a $G_{6}$ for the degenerate case $\omega=0$ [13]. There are three different classes of non-degenerate $(\omega \neq 0)$ Gödel-type metrics, according to the solutions of Eqs. (2), namely,

i) hyperbolic class, where $m^{2}>0$ and

$$
\begin{aligned}
& H(r)=\frac{2 \omega}{m^{2}}[\cosh (m r)-1], \\
& D(r)=\frac{1}{m} \sinh (m r) ;
\end{aligned}
$$

ii) trigonometric class, where $m^{2}=-\mu^{2}<0$ and

$$
\begin{aligned}
& H(r)=\frac{2 \omega}{\mu^{2}}[1-\cos (\mu r)], \\
& D(r)=\frac{1}{\mu} \sin (\mu r) ;
\end{aligned}
$$

iii) linear class, where $m^{2}=0$ and

$$
\begin{aligned}
& H(r)=\omega r^{2}, \\
& D(r)=r .
\end{aligned}
$$

The Gödel solution [12 belongs to the hyperbolic class in which $0 \leq m^{2}=2 \omega^{2}$. It is a solution of Einstein equations with the cosmological constant $\Lambda$ for a dust of density $\rho$ and rigid rotation $\omega$, such that $m^{2}=2 \omega^{2}=-2 \Lambda=\kappa \rho$, where $\kappa$ is the Einstein constant. But through the change of variables $\rho=p^{\prime}-\rho^{\prime}, \Lambda=\Lambda^{\prime}-\kappa p^{\prime}$ it can be reinterpreted as a solution with a cosmological constant $\Lambda^{\prime}$ and a perfect fluid with pressure $p^{\prime}$, density $\rho^{\prime}$ and vorticity $\omega$, where $m^{2}=2 \omega^{2}=\kappa\left(p^{\prime}+\rho^{\prime}\right)$ and $2 \Lambda^{\prime}=p^{\prime}-\rho^{\prime}$.

The closed time-like curves of Gödel-type space-times are circles $C=\{(t, r, \theta, z) ; t, r, z=$ const, $\theta \in[0,2 \pi]\}$, in a region defined by a range of $r\left(r_{1}<r<r_{2}\right)$ where $G(r)=D^{2}(r)-H^{2}(r)<$ 0 . For the linear class $m=0$, there is a non-causal region $r>r_{c}$ with closed time-like curves, where $r_{c}=1 / \omega$ is a critical radius. For the trigonometric class $m^{2}=-\mu^{2}<0$, there is an infinite sequence of alternating causal and non-causal regions. For the hyperbolic class $m^{2}>0$, when $0<m^{2}<4 \omega^{2}$ there is a non-causal region $r>r_{c}$, where the critical radius $r_{c}$ is given by

$$
\sinh ^{2}\left(\frac{m r_{c}}{2}\right)=\left(\frac{4 \omega^{2}}{m^{2}}-1\right)^{-1},
$$

and when $m^{2} \geq 4 \omega^{2}$ there is no breakdown of causality, since for $m^{2}=4 \omega^{2}$ the critical radius $r_{c} \rightarrow \infty$ [13]. There is no analogous situation for the linear and trigonometric classes, where there is no way to circumvent the violation of causality.

\section{GÖDEL-TYPE METRICS IN GR}

In this section we review the Gödel-type solutions of GR for the physically well-motivated matter content discussed in Refs. [13, having in mind our objective to investigate their possible 
extensions for the case of the CS modified gravity. In order to characterize completely the Gödeltype universes in GR, it is necessary the consider the matter sources of the Gödel-type metrics. Since these metrics are ST-homogeneous, there is a local Lorentz co-frame $w^{A}=e_{\mu}^{A} d x^{\mu}$ where the components of the curvature tensor are constants. It is given by [13

$$
\begin{aligned}
& w^{(0)}=d t+H(r) d \theta, \\
& w^{(1)}=d r, \\
& w^{(2)}=D(r) d \theta, \\
& w^{(3)}=d z,
\end{aligned}
$$

where $d s^{2}=\eta_{A B} w^{A} w^{B}$ and $\eta_{A B}=\operatorname{diag}(-1,+1,+1,+1)$ is the Minkowski metric. The Einstein field equations $R_{A B}-\frac{1}{2} \eta_{A B} R=\kappa T_{A B}-\eta_{A B} \Lambda$, in the Lorentz frame (7), can be written as

$$
R_{A B}=\kappa\left(T_{A B}-\frac{1}{2} \eta_{A B} T\right)+\Lambda \eta_{A B}
$$

where $T=\eta^{A B} T_{A B}$ is the trace of energy-momentum tensor of matter $T_{A B}$.

Our conventions for the curvature and Ricci tensors are $R_{\mu \beta \nu}^{\alpha}=\Gamma_{\beta \mu, \nu}^{\alpha}-\Gamma_{\mu \nu, \beta}^{\alpha}+\Gamma_{\rho \nu}^{\alpha} \Gamma_{\beta \mu}^{\rho}-\Gamma_{\rho \beta}^{\alpha} \Gamma_{\nu \mu}^{\rho}$ and $R_{\mu \nu}=R_{\mu \alpha \nu}^{\alpha}$. Small Greek letters label coordinate indices and capital Latin letters label Lorentz tetrad indices, and both take values from 0 to 3 .

For all three classes of Gödel-type metrics given by Eqs. (345), the curvature scalar is given by $R=2\left(\omega^{2}-m^{2}\right)$, and the non-vanishing components of Ricci tensor, in the Lorentz co-frame defined by Eqs. (7) above, are $R_{(0)(0)}=2 \omega^{2}, R_{(1)(1)}=R_{(2)(2)}=2 \omega^{2}-m^{2}$. Since $R_{A B}$ is constant and diagonal, it follows that $T_{A B}$ has to be constant and diagonal, due to the Einstein field equations. There is a preferred direction in Gödel-type space-times determined by the rotation vector which, for the Local Lorentz frame observers defined by Eqs. (7) and 4-velocity $u^{A}=e_{0}^{A}=\delta_{0}^{A}$, is given by $\omega^{A}=\frac{1}{2} \epsilon^{A B C D} u_{B} \omega_{C D}=[0,0,0,-\omega]$, where $\epsilon^{A B C D}=\frac{1}{\sqrt{-g}} \varepsilon^{A B C D}\left(\varepsilon^{0123}=1\right)$ is the totally antisymmetric Levi-Civita tensor, and $\omega_{A B}$ is the vorticity tensor.

In order to compare the results of GR and CS modified gravity, we use the same matter content of the universe considered in [13], that is, a perfect fluid, a scalar field and an electromagnetic field. Let us start with the perfect fluid, which has rigid rotation velocity $\omega$, density $\rho$ and pressure $p$, whose components of the energy-momentum tensor $T_{A B}^{(p f)}=(p+\rho) u_{A} u_{B}+p \eta_{A B}$, in the Lorentz tetrad basis defined by Eqs. (7), are

$$
T_{(0)(0)}^{(p f)}=\rho, T_{(1)(1)}^{(p f)}=T_{(2)(2)}^{(p f)}=T_{(3)(3)}^{(p f)}=p,
$$

where the 4 -velocity of an element of fluid is $u^{A}=e_{0}^{A}=\delta_{0}^{A}$. Notice that $T^{(\mathrm{pf})}{ }_{A B}$ is constant and diagonal. The next matter source is a massless scalar field $\psi$ satisfying the Klein-Gordon 
equation $\square \psi=\eta^{A B} \nabla_{A} \nabla_{B} \psi=0$. Following [13], we choose a scalar field whose gradient $\partial_{A} \psi=$ $e_{A}{ }^{\mu} \partial_{\mu} \psi$, in the Lorentz tetrad basis defined by Eqs. (7), is constant and parallel to $z$ axis. Thus, $\psi=s\left(z-z_{0}\right)$, where $s$ is a constant. The components of its energy-momentum tensor $T_{A B}^{(s f)}=$ $\partial_{A} \psi \partial_{B} \psi-\frac{1}{2} \eta_{A B} \eta^{C D} \partial_{C} \psi \partial_{D} \psi$, in the Lorentz co-frame given by Eqs. (7), are

$$
T_{(0)(0)}^{(s f)}=T_{(3)(3)}^{(s f)}=\frac{s^{2}}{2}, T_{(1)(1)}^{(s f)}=T_{(2)(2)}^{(s f)}=-\frac{s^{2}}{2} .
$$

Finally, the last source in [13] is a source-free electromagnetic field $F_{A B}$ that is a solution of the vacuum Maxwell equations $\nabla_{B} F^{A B}=0$ and $\nabla_{B}{ }^{*} F^{A B}=0$, where ${ }^{*} F^{A B}=\frac{1}{2} \epsilon^{A B C D} F_{C D}$. According to [13, let us choose an electromagnetic field $F_{A B}$ such that, in the Lorentz co-frame (7), both the electric and the magnetic fields are parallel to $z$ axis, that is, $F_{(0)(3)}=E(z)=e \sin \left[2 \omega\left(z-z_{0}\right)\right]$ and $F_{(1)(2)}=B(z)=-e \cos \left[2 \omega\left(z-z_{0}\right)\right]$, where $e$ is a constant. The non-vanishing components of the energy-momentum tensor $T_{A B}^{(e f)}=F_{A}{ }^{N} F_{B N}-\frac{1}{4} \eta_{A B} F^{C D} F_{C D}$ of the electromagnetic field, in the Lorentz co-frame (7), are given by

$$
T_{(0)(0)}^{(e f)}=T_{(1)(1)}^{(e f)}=T_{(2)(2)}^{(e f)}=\frac{e^{2}}{2}, T_{(3)(3)}^{(e f)}=-\frac{e^{2}}{2} .
$$

To resume, the energy-momentum tensor of all matter sources is given by

$$
T_{A B}=T_{A B}^{(p f)}+T_{A B}^{(s f)}+T_{A B}^{(e f)},
$$

which is diagonal with components constants. Thus, by taking the Einstein constant $\kappa=1$, the Einstein equations (8) for an arbitrary Gödel-type metric with parameters $\left(\omega, m^{2}\right)$, in the Lorentz tetrad basis (7), are given by

$$
\begin{aligned}
2 \omega^{2} & =\frac{1}{2} e^{2}+\frac{1}{2} \rho+\frac{3}{2} p-\Lambda \\
2 \omega^{2}-m^{2} & =\frac{1}{2} e^{2}-\frac{1}{2} p+\frac{1}{2} \rho+\Lambda \\
0 & =-\frac{1}{2} e^{2}+s^{2}-\frac{1}{2} p+\frac{1}{2} \rho+\Lambda .
\end{aligned}
$$

The cosmological constant is completely determined by the matter source, according to Eq. (15), and does not depend on the metric parameters $\left(\omega, m^{2}\right)$, since $R_{(3)(3)}=0$. Solving the field equations 1315 for the cosmological constant $\Lambda$ and the metric parameters $m^{2}$ and $\omega$, we obtain that

$$
\begin{aligned}
& 2 \omega^{2}=\rho+p+s^{2}, \\
& m^{2}=\rho+p+2 s^{2}-e^{2}, \\
& 2 \Lambda=-\rho+p-2 s^{2}+e^{2} .
\end{aligned}
$$


This matter content gives rise to solutions belonging to all three classes of Gödel-type metrics, but both the linear and trigonometric classes require the existence of the electromagnetic field, according to Eq. (17). Solutions for the hyperbolic class, where $m^{2}>0$, are obtained when $\rho+p+2 s^{2}-e^{2}>0$. There is an upper bound for $m^{2}$ given by $m^{2} \leq 4 \omega^{2}$, since

$$
m^{2}-4 \omega^{2}=-\left(p+\rho+e^{2}\right) \leq 0
$$

Therefore, there is no solution within the causal region, except the Rebouças-Tiomno solution, where $m^{2}=4 \omega^{2}$, for a matter described purely by the scalar field [13]. The Gödel solution $m^{2}=2 \omega^{2}=3 s^{2}+p+\rho-2 e^{2}>0$ is recovered either for all the sources, when $s^{2}=e^{2} \neq 0$, or for a pure perfect fluid, when $s^{2}=e^{2}=0$, since

$$
m^{2}-2 \omega^{2}=s^{2}-e^{2}
$$

Furthermore, when $s^{2} \leq e^{2}$, there is a lower upper bound for $m^{2}$ given by $m^{2} \leq 2 \omega^{2}$, and when $s^{2} \geq e^{2}$, there is a non-zero lower bound given by $2 \omega^{2} \leq m^{2}$.

The solutions for the linear class, where $m^{2}=0$, are obtained when $\rho+p+2 s^{2}-e^{2}=0$, and the solutions of the trigonometric class, where $m^{2}=-\mu^{2}<0$, are obtained when $\rho+p+2 s^{2}-e^{2}<0$. An important result is that the perfect fluid and the scalar field produce hyperbolic solutions within the interval $2 \omega^{2} \leq m^{2} \leq 4 \omega^{2}$.

The sign of the cosmological constant for all three classes can be easily determined from

$$
2 \Lambda=2 p-m^{2}
$$

which is obtained by summing of Eqs. (18) and (17). For the hyperbolic class, the cosmological constant is positive when $2 p>m^{2}>0$, negative when $0 \leq 2 p<m^{2}$ and zero when $0<2 p=m^{2}$. For the linear class, we have $\Lambda=p \geq 0$, since $m^{2}=0$, and for the trigonometric class, where $m^{2}=-\mu^{2}<0$, we have only $2 \Lambda=2 p+\mu^{2}>0$.

Note that the presence of the electromagnetic field is essential for the consistency of all three classes of solutions, in other words, in its absence, one has only the hyperbolic class, where all solutions are within the interval $0<m^{2}<4 \omega^{2}$ inside the non-causal region, except of a pure scalar field corresponding to the case $m^{2}=4 \omega^{2}$.

\section{GÖDEL-TYPE METRICS IN CHERN-SIMONS MODIFIED GRAVITY}

In this section, after a brief review of the non-dynamical CS modified gravity, we investigate the existence of Gödel-type ST-homogeneous solutions of the CS-modified gravitational field equations, 
as well as their causality features, in order to compare the GR and CS gravitational theories with respect to this kind of causality violations. In particular, since we are searching for generalizations of the Gödel-type solutions obtained in [13, the same matter content will be the source for the CS modified gravitational field.

The CS modified gravity action 3 , with the cosmological constant is

$$
S=\frac{1}{2 \kappa} \int d^{4} x\left[\sqrt{-g}(R-2 \Lambda)+\frac{1}{4} \phi^{*} R R\right]+S_{m},
$$

where $\phi$ is a scalar field, $S_{m}$ is the matter action and ${ }^{*} R R$ is the Pontryagin density defined by

$$
{ }^{*} R R \equiv{ }^{*} R_{B}^{A}{ }_{B}^{C D} R_{A C D}^{B}=\frac{1}{2} \varepsilon^{C D E F} R_{B E F}^{A} R_{A C D}^{B} .
$$

The scalar field $\phi$ is called the CS coefficient, or CS coupling field, and measures how the modified theory deforms the GR.

The CS gravitational field equations are obtained by varying the CS modified action with respect to the metric $g_{\mu \nu}$ and the CS coupling field $\phi$ and, in the Lorentz tetrad basis defined by Eqs. (7), they can be written as

$$
\begin{aligned}
R_{A B}+C_{A B} & =\kappa\left(T_{A B}-\frac{1}{2} \eta_{A B} T\right)+\eta_{A B} \Lambda, \\
{ }^{*} R R & =0
\end{aligned}
$$

where $T$ is the trace of the matter energy-momentum tensor $T^{A B}$, and $C^{A B}$ is the Cotton tensor arising due to variation of the CS term, defined as [3]:

$$
\begin{aligned}
C^{A B}= & -\frac{1}{2}\left[\left(\epsilon^{C A D E} \nabla_{D} R_{E}^{B}+\epsilon^{C B D E} \nabla_{D} R_{E}^{A}\right) \partial_{C} \phi\right. \\
& \left.+\left({ }^{*} R^{E A F B}+{ }^{*} R^{E B F A}\right) \nabla_{E} \nabla_{F} \phi\right] .
\end{aligned}
$$

The Eq. (25) means that the theory involves a constraint, i.e., such a field equation imposes restrictions on the possible space-time geometries. Furthermore, the covariant divergence of the Cotton tensor is

$$
\nabla_{A} C^{A B}=\frac{1}{8 \sqrt{-g}}{ }^{*} R R \partial^{B} \phi,
$$

that implies, by Eqs. (24), which the covariant divergence of $T_{A B}$ is proportional to ${ }^{*} R R$, in other words, the Pontryagin density measures the rate of breaking of the diffeomorphism invariance.

We shall study the Gödel-type metrics in the CS modified gravity context and investigate the causality features, as well as the possible simultaneous solutions among GR and the CS modified 
gravity. For this, we shall consider the same matter sources used in the paper [13] and combinations between them as particular cases.

In order to calculate the CS gravitational field equations for a Gödel-type metric, it is necessary to calculate first the Cotton tensor given by Eqs. (26) and the Pontryagin scalar density given by Eq. (23). For an arbitrary CS coupling field $\phi(t, r, z)$ with a cylindrical symmetry and an arbitrary Gödel-type metric with components $H(r)$ and $D(r)$, we obtain that ${ }^{*} R R=0$, and that the non-vanishing components of the Cotton tensor are given by the diagonal components

$$
C_{(0)(0)}=2 C_{(1)(1)}=2 C_{(2)(2)}=2 \frac{\partial \phi}{\partial z} \omega\left(4 \omega^{2}-m^{2}\right),
$$

and the non-diagonal components

$$
\begin{aligned}
& C_{(0)(1)}=-\frac{1}{2} \frac{\partial^{2} \phi}{\partial z \partial t} \frac{H(r)}{D(r)}\left(4 \omega^{2}-m^{2}\right) \\
& C_{(0)(2)}=-\frac{1}{2} \frac{\partial^{2} \phi}{\partial z \partial r}\left(4 \omega^{2}-m^{2}\right) \\
& C_{(0)(3)}=-\frac{\partial \phi}{\partial t} \omega\left(4 \omega^{2}-m^{2}\right) \\
& C_{(1)(3)}=-\frac{1}{2} \frac{\partial^{2} \phi}{\partial t^{2}} \frac{H(r)}{D(r)}\left(4 \omega^{2}-m^{2}\right) \\
& C_{(2)(3)}=\frac{1}{2} \frac{\partial^{2} \phi}{\partial t \partial r}\left(4 \omega^{2}-m^{2}\right) .
\end{aligned}
$$

The Rebouças-Tiomno Gödel-type universe, corresponding to $4 \omega^{2}=m^{2}$, solves the CS gravitational field equations trivially, since it follows from Eqs. $(28+29)$ that in this case $C_{A B}=0$, for an arbitrary CS coupling field $\phi$. Therefore, for $4 \omega^{2}=m^{2}$ the CS field equations are identical to Einstein field equations, and we must require that $4 \omega^{2} \neq m^{2}$ in order to obtain nontrivial solutions of the CS modified gravitational field equations.

Taking into account that for an arbitrary ST-homogeneous Gödel-type metric the Ricci tensor is diagonal, and that the matter source considered in [13] is also diagonal in the Lorentz tetrad basis defined by Eqs. (7), one concludes that the non-diagonal elements of the Cotton tensor 29 should vanish. Therefore, we have the equations $C_{A B}=0$ (for $A \neq B$ ), whose solution is $\phi(t, r, z)=b\left(z-z_{0}\right)+f(r)$, where $b$ and $z_{0}$ are arbitrary constants and $f(r)$ is an arbitrary function. Since the diagonal components of the Cotton tensor 28 involve only the derivatives $\frac{\partial \phi}{\partial z}$, we can choose $f(r)=0$ for the sake of simplicity without loss of generality. Therefore the CS coupling field is restricted to be

$$
\phi(z)=b\left(z-z_{0}\right)
$$


whose gradient $\partial_{A} \phi=[0,0,0, b]$ is parallel to the vorticity vector $\omega_{A}=[0,0,0,-\omega]$ and aligned with the $z$ axis, the preferred direction of the ST-homogeneous Gödel-type metrics. This allows us to introduce a new arbitrary parameter $k$ defined by the inner product $\omega^{A} \partial_{A} \phi=-b \omega=-k$. Both the gradient $\partial_{A} \phi$ of the CS scalar field and the vorticity $\omega^{A}$ have the same direction and sense when $k<0$. Thus, since there is a coupling between the vorticity and the CS scalar field, we can choose either $b$ or $k$ as an independent parameter. As we will see later, it is better to consider

$$
b=\frac{k}{\omega}
$$

In order to compare the results of GR and CS gravity, we continue to use the matter content of the Gödel-type solutions of GR obtained in [13], whose energy-momentum tensor is given by Eqs. (12), as well the CS modified gravitational field equations (24). Thus, for the CS scalar field given by Eq. (30) and an arbitrary Gödel-type metric with components $H(r)$ and $D(r)$, in the Lorentz tetrad basis defined by Eqs. (7), we obtain the following form of the CS modified gravitational field equations

$$
\begin{aligned}
2 \omega^{2}+2 b \omega\left(4 \omega^{2}-m^{2}\right) & =\frac{1}{2} e^{2}+\frac{1}{2} \rho-\Lambda+\frac{3}{2} p, \\
2 \omega^{2}-m^{2}+b \omega\left(4 \omega^{2}-m^{2}\right) & =\frac{1}{2} e^{2}-\frac{1}{2} p+\Lambda+\frac{1}{2} \rho, \\
0 & =-\frac{1}{2} e^{2}-\frac{1}{2} p+s^{2}+\Lambda+\frac{1}{2} \rho,
\end{aligned}
$$

with the requirement that $4 \omega^{2} \neq m^{2}$, in order to have $C_{A B} \neq 0$ for an arbitrary CS coefficient.

In CS modified gravity the cosmological constant $\Lambda$ is related only to the matter content and is the same for both GR and CS solutions, given by Eq. (18), that is, $2 \Lambda=-\rho+p-2 s^{2}+e^{2}$, since the CS field equation (34) is equal to the equation (15). The remaining two CS gravitational equations 32 33 can be used to determine the metric parameters $\left(\omega, m^{2}\right)$ in terms of the matter content and the CS scalar field, where $b$ is considered as an arbitrary parameter, since there are three parameters and two equations. Following this procedure, expressing $m^{2}$ from (33) and substituting in Eq. (32) we arrive at a third order algebraic equation for $\omega$. On the other hand, taking into account that the CS scalar field parameter $b$ appears in the CS gravitational equations only through the combination $b \omega$, we substitute Eq. (31) in the equations for CS gravitational field 32 33). Treating $k$ as an arbitrary parameter, we obtain linear equations with respect to both $m^{2}$ and $\omega^{2}$ which, after substituting $\Lambda$ through 18 , are given by

$$
\begin{aligned}
(2+8 k) \omega^{2}-2 k m^{2} & =\rho+s^{2}+p \\
(2+4 k) \omega^{2}-(1+k) m^{2} & =-s^{2}+e^{2} .
\end{aligned}
$$


Although there is no Gödel-type vacuum solution in GR, the CS modified field equations 355 have a vacuum solution given by

$$
\begin{aligned}
\Lambda & =0, \\
m^{2} & =\omega^{2}, \\
b & =\frac{k}{\omega}=-\frac{1}{3 \omega}
\end{aligned}
$$

This solution belongs to the hyperbolic class and presents causal anomalies, since $0<m^{2}<4 \omega^{2}$, therefore we see that the causality violation can occur without any matter, only due to the CS coefficient itself which can be treated as a some special non-dynamical media. Even being nondynamical, the CS field $\phi$ interacts with the metric in such a way that the vacuum CS field equations give rise to a non-trivial space-time geometry, where the non-vanishing components of the Ricci tensor are $R_{(0)(0)}=2 R_{(1)(1)}=2 R_{(2)(2)}=2 \omega$ and the curvature scalar is $R=0$.

On the other hand, the non-vacuum solution of the CS field equations $35 \sqrt[36]{36}$ for $\omega^{2}(k)$ is given by

$$
\omega^{2}(k)=\frac{a_{\omega} k+b_{\omega}}{3 k+1}=\frac{1}{2} \frac{\left(\rho+p+3 s^{2}-2 e^{2}\right) k+\rho+p+s^{2}}{3 k+1},
$$

for $k \neq-\frac{1}{3}$, whose coefficients

$$
\begin{aligned}
& 2 a_{\omega}=\rho+p+3 s^{2}-2 e^{2}, \\
& 2 b_{\omega}=\rho+p+s^{2},
\end{aligned}
$$

are determined only by the matter content. Similarly the solution for $m^{2}(k)$ is given by

$$
m^{2}(k)=\frac{a_{m} k+b_{m}}{3 k+1}=\frac{2\left(\rho+p+3 s^{2}-2 e^{2}\right) k+\rho+p+2 s^{2}-e^{2}}{3 k+1},
$$

for $k \neq-\frac{1}{3}$, with coefficients

$$
\begin{aligned}
& a_{m}=2\left(\rho+p+3 s^{2}-2 e^{2}\right), \\
& b_{m}=\rho+p+2 s^{2}-e^{2} .
\end{aligned}
$$

The signs of the metric parameters $\omega^{2}$ and $m^{2}$ given by the GR solution 16 18, depend on the matter content and on the range of $k$. Since $\omega$ is real, we must impose the condition $\omega^{2}(k)>0$, which determines the allowed range of $k$ and, in turn, also determine the possible signs of $m^{2}(k)$ and the classes of the Gödel-type metric within the allowed range.

When the CS coefficient vanishes, i.e. $k=0$, or, as is the same, $b=0$, see (31), the CS solution given by (38 41) reduces to $\omega^{2}=b_{\omega}$ and $m^{2}=b_{m}$, thus reproducing the GR solution 16 18. In 
other words, the coefficients $b_{\omega}$ and $b_{m}$ of the CS solution are reduced to the GR results for $\omega^{2}$ and $m^{2}$, respectively, for the same matter content.

The causality features of the CS solution $(38,41)$ is determined by the sign of

$$
m^{2}-4 \omega^{2}=\frac{-\left(\rho+p+e^{2}\right)}{3 k+1},
$$

which depends on the allowed range of $k$. In general, for $k<-\frac{1}{3}$ we have $m^{2}>4 \omega^{2}$ and the solution is causal, while for $k>-\frac{1}{3}$ we have $m^{2}<4 \omega^{2}$ and the solution is non-causal. And for $k=-\frac{1}{3}$, we have the vacuum solution which is non-causal.

There are general relations between the coefficients of $\omega^{2}$ and $m^{2}$ above, given by $a_{m}=4 a_{\omega}$ and $b_{m}=b_{\omega}+a_{\omega}$. The root $k_{\omega}=-\frac{b_{\omega}}{a_{\omega}}$ of $\omega^{2}(k)$ and the root $k_{m}=-\frac{b_{m}}{a_{m}}$ of $m^{2}(k)$ are also related as $k_{\omega}=4 k_{m}+1$. There are also the restrictions $a_{\omega}-3 b_{\omega}=-\rho-p-e^{2} \leq 0$ and $a_{m}-3 b_{m}=-\rho-p-e^{2} \leq 0$. Using the conditions $a_{\omega}=3 b_{\omega}$ and $a_{m}=3 b_{m}$ in the CS solution, which takes place only for a pure scalar field source $\psi=s\left(z-z_{0}\right)$, we obtain that $\omega^{2}(k)=b_{\omega}=\frac{1}{2} s^{2}$ and $m^{2}(k)=b_{m}=2 s^{2}$ (for any $k$ ). This is the Rebouças-Tiomno GR solution [13, where $m^{2}=4 \omega^{2}$, for which the Cotton tensor vanishes, independently of the CS scalar field. Thus, differently from GR, CS gravity has only a trivial solution in presence of a pure scalar field.

There is a special CS solution obtained when the matter source satisfies the conditions $2 a_{\omega}=$ $\rho+p+3 s^{2}-2 e^{2}>0$ and $2 b_{\omega}=\rho+p+s^{2}>0$, since these conditions imply that both $a_{m}>0$ and $b_{m}>0$ and the roots of $\omega^{2}(k)$ and $m^{2}(k)$ are related through the relation $k_{\omega}=-\frac{b_{\omega}}{a_{\omega}}<k_{m}=$ $-\frac{b_{m}}{a_{m}}<-\frac{1}{3}$. The main features of this solution is most evident when the source is given by a pure dust, so that CS solution (38,41) can be written as

$$
\begin{aligned}
\frac{\omega^{2}(k)}{\rho} & =\frac{1}{2} \frac{k+1}{3 k+1}, \\
\frac{m^{2}(k)}{\rho} & =\frac{2 k+1}{3 k+1},
\end{aligned}
$$

whose roots are $k_{\omega}=-1$ and $k_{m}=-1 / 2$, respectively. This dust solution must satisfy the requirement $\omega^{2}(k)>0$, since $\omega$ is real, which determines the allowed range of $k$ given by $k<-1$ or $k>-1 / 3$, where $m^{2}(k)>0$ as well. This can be easily seen in Fig. (1), with the graphics of the $\omega(k)>0$ and $m^{2}(k)$, are given for $\rho=1$. Therefore, the Gödel-type metric of the CS dust solution above is hyperbolic and has a GR analogue, since $k=0$ belongs to the allowed range. Treating the causality, for $k<-1$ the space-time is causal and for $k>-1 / 3$ the space-time is non-causal. A distinguishing feature of CS gravity, contrasting to GR, is the existence of the hyperbolic causal solutions for physically well-motivated matter sources, whose energy-momentum tensor given by Eq. 12 , where $\rho+p+3 s^{2}-2 e^{2}>0$. 


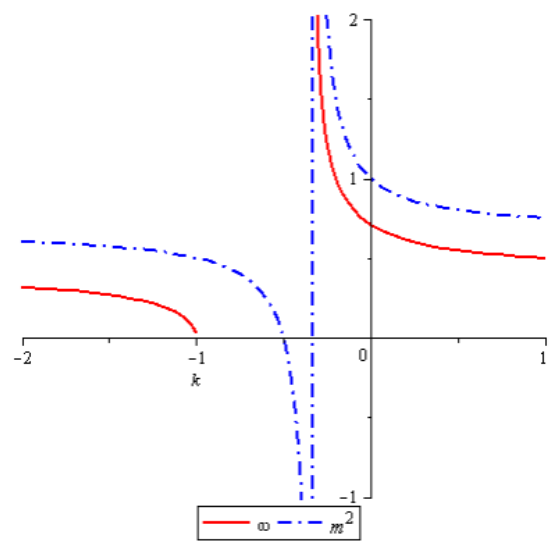

Figure 1: CS solution $\omega(k)$ and $m^{2}(k)$ for dust with $\rho=1$.

The Gödel metric solution of CS gravity can be obtained by imposing, for the CS solution 38 (31) the following condition

$$
m^{2}-2 \omega^{2}=-\frac{\left(-3 s^{2}-p-\rho+2 e^{2}\right) k+e^{2}-s^{2}}{3 k+1}=0,
$$

whose result can be presented as

$$
\begin{aligned}
m^{2} & =2 \omega^{2}=3 s^{2}+p+\rho-2 e^{2}>0, \\
b \omega & =k=\frac{e^{2}-s^{2}}{2 \omega^{2}} \neq 0 .
\end{aligned}
$$

Therefore, it is insufficient to have a pure perfect fluid to achieve this solution, as happens in GR, since the condition $b \neq 0$ requires the presence either of an electromagnetic field or of a scalar field, according to Eq. (49). On the other hand, the matter source can also be given by an electromagnetic field and a scalar field, where $e^{2}-s^{2} \neq 0$. This is an extension of the GR solution, which is recovered when $e^{2}-s^{2}=0$.

Now let us discuss the existence of the linear and trigonometric classes in CS modified gravity. Without the electromagnetic field, all solutions have the same behaviour as the dust solution 45 . 46), where all coefficients $a_{\omega}, b_{\omega}, a_{m}$ and $b_{m}$ are positive, belonging only to the hyperbolic class. Therefore, similarly to GR, the presence of the electromagnetic field is necessary in order to obtain solutions of linear and trigonometric classes. Although the GR solution given by Eqs. 16, 18, for the non-degenerate case where $\omega \neq 0$, cannot be achieved if the source is presented by a pure electromagnetic field, for the CS solution given by Eqs. (38) and Eq. (18), this is not only possible, but also all the classes are obtained.

The CS solution given by Eq. (18) and Eqs. (38,41), when the source is a pure electromagnetic 
field, is given by $2 \Lambda=e^{2}$ and

$$
\begin{aligned}
\frac{\omega^{2}}{e^{2}} & =-\frac{k}{3 k+1}, \\
\frac{m^{2}}{e^{2}} & =-\frac{4 k+1}{3 k+1},
\end{aligned}
$$

respectively. The roots of $\omega^{2}$ and $m^{2}$ are $k_{\omega}=0$ and $k_{m}=-1 / 4$, correspondingly, and do not depend on the value of $e^{2}$. This solution must satisfy the requirement $\omega^{2}(k)>0$, since $\omega$ is real. It follows that the allowed range of $k$ is given by $-1 / 3<k<0$ and that the solution given by the Eqs. 50 51) is hyperbolic $\left(m^{2}>0\right)$ for $-1 / 3<k<-1 / 4$, linear $\left(m^{2}=0\right)$ for $k_{m}=-1 / 4$ and trigonometric $\left(m^{2}<0\right)$ for $-1 / 4<k<0$. These results can be seen in Fig. 2, with the graphics of $\omega(k)$ and $m^{2}(k)$ are given for $e^{2}=1$, which shows the main features of the pure electromagnetic CS solution. The hyperbolic solution is non-causal, since $\omega^{2}>m^{2}>0$ for $-1 / 3<k<0$, that is, in all allowed range of $k$. All of these solutions have no analogue in GR.

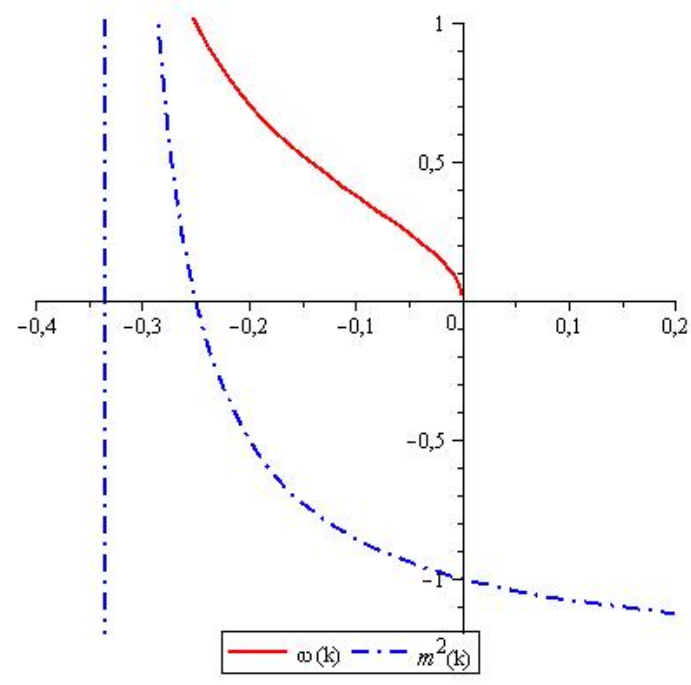

Figure 2: $w$ and $m^{2}$, for $e=1$

\section{SUMMARY}

We discussed the consistency of the Gödel-type metric and the possibility of existence of closed time-like curves within the CS modified gravity. Within our studies, we used a tetrad basis and a tetrad formalism allowing to simplify the calculations crucially, and considered the metric corresponding to a non-zero Cotton tensor, differently from [14] where the consistency of the Gödel metric has been verified only in the case of the vanishing Cotton tensor. We considered all three 
characteristic forms of a generic Gödel-type metric, including not only hyperbolic case but also trigonometric and linear cases, and a rather generic form of the matter, involving a relativistic fluid, a cosmological constant, scalar and electromagnetic fields.

We found that the Gödel-type metric indeed solves the modified Einstein equations in certain cases. The most interesting among them is certainly the hyperbolic case which is reproduced if the CS term is switched off. We also found that in certain cases we can have solutions consistent with existence of CTCs. We found that unlike the GR, in the CS modified gravity there is a nontrivial solution for the vacuum case. Also, we found several other solutions having no analogues in the GR case, with the most important among them is the hyperbolic causal solution.

We demonstrated that the GR limit in the CS modified gravity is recovered by making the amplitude of CS coefficient equal to zero, $b=0$. Furthermore, due to arbitrariness of parameter $b$, it opened up a wide range of the Gödel-type possible solutions, among which we obtained some hyperbolic causal solutions whose existence is impossible in GR, except of pure scalar field case. We found that the presence of the electromagnetic field plays the crucial role for linear and trigonometric solutions, just as in the usual GR case. Thus, we conclude that the impact of the CS extension becomes fundamental for the causality features for all relevant matter sources. We note that arising of all these solutions does not require any presence of the exotic matter.

Acknowledgments. Authors are grateful to M. Rebouças for important discussions. This work was partially supported by Conselho Nacional de Desenvolvimento Científico e Tecnológico (CNPq). The work by A. Yu. P. has been supported by the CNPq project No. 303783/2015-0. The work by A. F. S. is supported by CNPq projects 476166/2013-6 and 201273/2015-2.

[1] S. Deser, R. Jackiw, S. Templeton, Ann. Phys. 140, 372 (1982).

[2] L. Alvarez-Gaume, E. Witten, Nucl. Phys. B234, 269 (1984).

[3] R. Jackiw, S.-Y. Pi, Phys. Rev. 68, 104012 (2003), gr-qc/0308071.

[4] V. A. Kostelecky, Phys. Rev. D69, 105009 (2004), gr-qc/0312310.

[5] T. Mariz, J. R. Nascimento, E. Passos, R. F. Ribeiro, Phys. Rev. D70, 024014 (2004), hep-th/0403205, T. Mariz, J. R. Nascimento, A. Yu. Petrov, L. Y. Santos, A. J. da Silva, Phys. Lett. B661, 312 (2008), arXiv: 0708.3348; M. Gomes, T. Mariz, J. R. Nascimento, A. Yu. Petrov, E. Passos, A. J. da Silva, Phys. Rev. D78, 025028 (2008), arXiv: 0805.4409; J. C. C. Felipe, A. R. Vieira, A. L. Cherchiglia, A. P. Baeta Scarpelli, M. Sampaio, Phys. Rev. D89, 105034 (2014), arXiv: 1403.2690.

[6] F. Izaurieta, E. Rodrigues, P. Salgado, "On transgression forms and Chern-Simons (super)gravity", hepth/0512014 F. Izaurieta, E. Rodrigues, P. Minning, P. Salgado, A. Perez, Phys. Lett. B678, 213 (2009), 
arXiv: 0905.2187; N. Merino, A. Perez, P. Salgado, Phys. Lett. B681, 85 (2009), arXiv: 0910.1470; F. Gomez, P. Minning, P. Salgado, Phys.Rev. D84, 063506 (2011).

[7] J. Zanelli, Lecture notes on Chern-Simons (super-)gravities, hep-th/0502193.

[8] S. Alexander, N. Yunes, Phys. Rept. 480, 1 (2009), arXiv: 0907.2562.

[9] D. Grumiller, N. Yunes, Phys. Rev. D77, 044015 (2008), arXiv: 0711.1868.

[10] K. Konno, T. Matsuyama, S. Tanda, Progr. Theor. Phys. 122, 561 (2009), arXiv: 0902.4767; N. Yunes, F. Pretorius, Phys. Rev. D79, 084043 (2009), arXiv: 0902.4669.

[11] M. Satoh, S. Kanno, J. Soda, Phys. Rev. D77, 023526 (2008), arXiv: 0706.3585; M. Satoh, J. Soda, JCAP 0809, 019 (2008), arXiv: 0806.4594.

[12] K. Godel, Rev. Mod. Phys. 21, 447 (1949).

[13] M. J. Rebouças, J. Tiomno, Phys. Rev. D28, 1251 (1983), Nuovo Cim. B90, 204 (1985); A. F. F. Teixeira, M. Rebouças, J. Aman, Phys. Rev. D32, 3309 (1985), J. Math. Phys. 27, 1370 (1986); M. O. Calvao, M. Rebouças, A. F. F. Teixeira, W. M. Silva, J. Math. Phys. 29, 1127 (1988); M. O. Calvao, I. D. Soares, J. Tiomno, Gen. Rel. Grav. 22, 683 (1990); H. L. Carrion, M. Rebouças, A. F. F. Teixeira, J. Math. Phys. 40, 4011 (1999), gr-qc/9904074, M. J. Rebouças, J. Santos, Phys. Rev. D80, 063009 (2009), arXiv: 0906.5354; J. Santos, M. Rebouças, T. B. R. F. Oliveira, Phys. Rev. 81, 123017 (2010), arXiv: 1004.2501; J. B. Fonseca-Neto, A. Yu. Petrov, M. Rebouças, Phys. Lett. B725, 412 (2013), arXiv: 1304.4675 .

[14] C. Furtado, T. Mariz, J. R. Nascimento, A. Yu. Petrov, A. F. Santos, Phys. Rev. D79, 124039 (2009), arXiv: 0906.0554; C. Furtado, J. R. Nascimento, A. Yu. Petrov, A. F. Santos, Phys. Lett. B693, 494 (2010), arXiv: 1004.5106. 